\title{
Complexity in Manufacturing Processes and Systems
}

\author{
Rosario Domingo $\mathbb{D},{ }^{1}$ Julio Blanco-Fernández $\mathbb{D}^{2}{ }^{2}$ \\ Jorge Luis García-Alcaraz $\mathbb{D}^{\mathbb{B}},{ }^{3}$ and Leonardo Rivera ${ }^{4}$ \\ ${ }^{1}$ Universidad Nacional de Educación a Distancia (UNED), Madrid, Spain \\ ${ }^{2}$ Universidad de La Rioja, Logroño, Spain \\ ${ }^{3}$ Autonomous University of Ciudad Juárez, Ciudad Juárez, CHIH, Mexico \\ ${ }^{4}$ Universidad del Valle, Cali, Colombia \\ Correspondence should be addressed to Rosario Domingo; rdomingo@ind.uned.es
}

Received 12 April 2018; Accepted 15 April 2018; Published 25 June 2018

Copyright ( 2018 Rosario Domingo et al. This is an open access article distributed under the Creative Commons Attribution License, which permits unrestricted use, distribution, and reproduction in any medium, provided the original work is properly cited.

The performance of activities in manufacturing systems and processes requires many factors that interact between them. Thus, machines, tooling, operators, techniques, methodologies, and information integrate a set that must be coordinated to work towards a common aim in an industrial plant. Some cases can be clearly observed in industrial plants as maquiladoras [1] or papermaking [2] where the operative parameters of the machines condition the final results, including the external factors as suppliers [3] and customers. Moreover, these interactions increase in a globalized market that demands a higher products variety with quality and on time. In this environment and under uncertainty, the complexity of the plant must be managed for integrating all their components. Efthymiou et al. [4] collected methods to manage the complexity as chaos and nonlinear dynamics theory, information theory, or hybrid procedures. These techniques are extended by Monostori [5] who also considers artificial intelligent and machine learning techniques; its application in manufacturing phases, as maintenance, allows controlling the process and predicting future behaviors [6].

In this context, proposals that allow controlling complexity or providing information to facilitate decision-making in environments where many factors interact under uncertainty are necessary and this special issue is aimed to contribute in that research area.

After a rigorous review process, nine papers have been accepted for this special issue. Among them, there are papers that present methods to reduce the uncertainty of the variables through the control of manufacturing parameters and others are focused to understand the production system and forecast scenarios.

G. Beruvides et al. focus the paper title "Coping with Complexity When Predicting Surface Roughness in Milling Processes: Hybrid Incremental Model with Optimal Parametrization" on the complexity of manufacturing processes, in particular in the surface roughness prediction during the milling by means of online efficient computational method. This work develops a method based on simulated annealing for optimal parameters tuning of the hybrid incremental model. This method provides better results regarding the accuracy and overall quality than traditional techniques (theoretical model, energy model, and Taguchi-based model) and other artificial intelligence-based techniques as Bayesian networks and multilayer perceptron for correctly predicting the surface roughness.

Z. Wang et al. in "Data-Driven Model-Free Adaptive Control of Particle Quality in Drug Development Phase of Spray Fluidized-Bed Granulation Process" propose a datadriven model-free adaptive control (DDMFAC) approach showing the algorithm stability and asymptotical convergence of tracking error. The model combines the principles of two types of control, the model-free adaptive control and data-driven optimal iterative learning control, and besides it adjusts the factor with fuzzy logic in order to adjust weighting factors. This approach is applied to a very important process of pharmaceutic industry, the control of particle quality in 
drug development phase of spray fluidized-bed granulation process, showing its effectiveness and better control than other approaches.

The paper titled "Research on the Complexity and Chaos Control about a Closed-Loop Supply Chain with DualChannel Recycling and Uncertain Consumer Perception" written by J. Ma et al. develops a closed-loop supply chain model with dual-channel recycling based on the game theory, the chaotic dynamics theory, and the complexity. The authors assume that the consumer perception regarding the remanufacturing products is uncertain. The dynamic game model is analyzed to keep the stable system. Numerical simulations are carried out to study the dynamic characteristics of the system by bifurcation diagram, largest Lyapunov exponent, and chaotic attractors, and the information entropy. Moreover, the authors introduce an efficient method to control chaos. The chaotic system can be delayed or eliminated adding an adjustment parameter regarding cooperation between the manufacturer and the third-party.

In the paper titled "Reliability Assessment Methodology for Massive Manufacturing Using Multi-Function Equipment," M. López-Campos et al. present a methodology for the reliability analysis of multifunction processes, using the Reliability Centered in Maintenance (RCM) approach, and a modification of the Universal Generating Function (UGF) in a multifunction manufacturing system. UGF is used in data preprocessing and analysis to determine the reliability of the multistate system. A case study focused on a plant of automated textile production is developed, considering different scenarios. With this methodology, the failure behavior of the entire workshop according to the scheduled jobs can be determined and the criticality of equipment elements can be identified.

The second group of papers is addressed towards the decision-making, presenting new or adapted methodologies, based on mainly multiobjective or multicriteria methods. They are presented in the following five paragraphs.

The paper "MOORA under Pythagorean Fuzzy Set for Multiple Criteria Decision Making," written by L. PérezDomínguez et al., presents a hybrid method (PF-MOORA) based on the multiobjective optimization on the basis of ratio analysis (MOORA) and Pythagorean fuzzy sets for multiple criteria decision-making. Two numerical cases are presented to assess the proposed hybrid methodology. These cases are focused on an assembly plant and on a mechanical manufacturing plant. Moreover, this method is compared with others and the results show its effectiveness and its similitude with the Pythagorean fuzzy TOPSIS (PF-TOPSIS), a technique for order of preference by similarity to ideal solution, in the assignment of the ranking of the best alternative.

J. Claver et al., in their paper titled "Decision-Making Methodologies for Reuse of Industrial Assets," develop tools for decision-making in the field of industrial assets. Multicriteria Decision Support Techniques are evaluated and the Analytic Hierarchy Process is adapted to develop methodologies for assessing both the heritage value and the most compatible uses according to the characteristics of the asset. The direct and indirect compatibilities are analyzed and applied to a case study, the warehouses of the Richard Gans Printing Works in Madrid, showing the good fitness between the methodology and the obtained results.

A. Realyvásquez and A. A. Maldonado-Macías in their paper titled "Measuring the Complex Construct of Macroergonomic Compatibility: A Manufacturing System Case Study" present a study that determines the levels of macroergonomic compatibility in a manufacturing system by means of a macroergonomic compatibility index (MCI) and a macroergonomic compatibility questionnaire. The authors find that the MCI can be an effective measurement of the factors and elements that interact with human resources. The MCI results show a medium level of macroergonomic compatibility of education, knowledge, and skills, which imply that the company considers employee educational characteristics and abilities in assigning workers to a particular job, but the company rarely considers other aspects, such as employee physical characteristics and work experience.

M.-S. Casas-Ramírez et al. in their paper titled "Optimizing a Biobjective Production-Distribution Planning Problem Using a GRASP" present a biobjective productiondistribution planning problem and an adapted Greedy Randomized Adaptive Search Procedure (GRASP) to minimize the total costs and to balance the total workload of the supply chain. The heuristic approach allows obtaining an approximation of the Pareto front. In this work, the Pareto front does not allow covering all the solutions space and it is discontinuous and nonconvex due to the characteristics of the defined model.

The paper titled "Modeling of Throughput in Production Lines Using Response Surface Methodology and Artificial Neural Networks" written by F. Nuñez-Piña et al. analyzes the NP-hard combinatorial problem of assigning buffers in a production line, considering the relationship between the number of buffer slots, the number of work stations, and the production rate. The authors develop two predictive models, which validate with data from the academic literature. The first is a fourth-order model based on response surface methodology (RSM) that presents a good fit with a coefficient of correlation close to 1 . The second is based on the artificial neural network (from 1 to 4 hidden layers and 5, 8, 10, 12, or 15 neurons for each layer), finding that the latter presents a better fit than the one developed by RMS, although both models have a good performance in predicting the throughput at short time.

The guest editors think that this special issue can be interesting for engineers and managers of manufacturing plants because the methods shown can facilitate the reduction of complexity and the decision-making. In addition, it can be interesting for researchers due to the relevance of this field and for some new exposed approaches.

\section{Acknowledgments}

The Guest Editorial Team would like to thank all the authors for their interest in selecting this special issue and for their valuable contributions. The editors would also like to thank all the anonymous reviewers who have contributed to improve the papers. 


\author{
Rosario Domingo \\ Julio Blanco-Fernández \\ Jorge Luis García-Alcaraz \\ Leonardo Rivera
}

\title{
References
}

[1] J. R. Díaz-Reza, J. L. García-Alcaraz, J. R. Mendoza-Fong, V. Martínez-Loya, E. J. Macías, and J. Blanco-Fernández, "Interrelations among SMED Stages: A causal model," Complexity, vol. 2017, pp. 1-10, 2017.

[2] L. M. Calvo and R. Domingo, "Influence of process operating parameters on $\mathrm{CO}_{2}$ emissions in continuous industrial plants," Journal of Cleaner Production, vol. 96, article no. 4308, pp. 253262, 2015.

[3] R. Villanueva-Ponce, J. L. Garcia-Alcaraz, G. Cortes-Robles, J. Romero-Gonzalez, E. Jiménez-Macías, and J. BlancoFernández, "Impact of suppliers' green attributes in corporate image and financial profit: case maquiladora industry," The International Journal of Advanced Manufacturing Technology, vol. 80, no. 5-8, pp. 1277-1296, 2015.

[4] K. Efthymiou, D. Mourtzis, A. Pagoropoulos, N. Papakostas, and G. Chryssolouris, "Manufacturing systems complexity analysis methods review," International Journal of Computer Integrated Manufacturing, vol. 29, no. 9, pp. 1025-1044, 2016.

[5] L. Monostori, "AI and machine learning techniques for managing complexity, changes and uncertainties in manufacturing," Engineering Applications of Artificial Intelligence, vol. 16, no. 4, pp. 277-291, 2003.

[6] N. Rodríguez-Padial, M. Marín, and R. Domingo, “An approach to integrating tactical decision-making in industrial maintenance balance scorecards using principal components analysis and machine learning," Complexity, vol. 2017, pp. 1-15, 2017. 


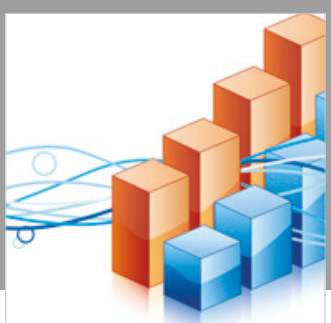

Advances in

Operations Research

\section{-n-m}
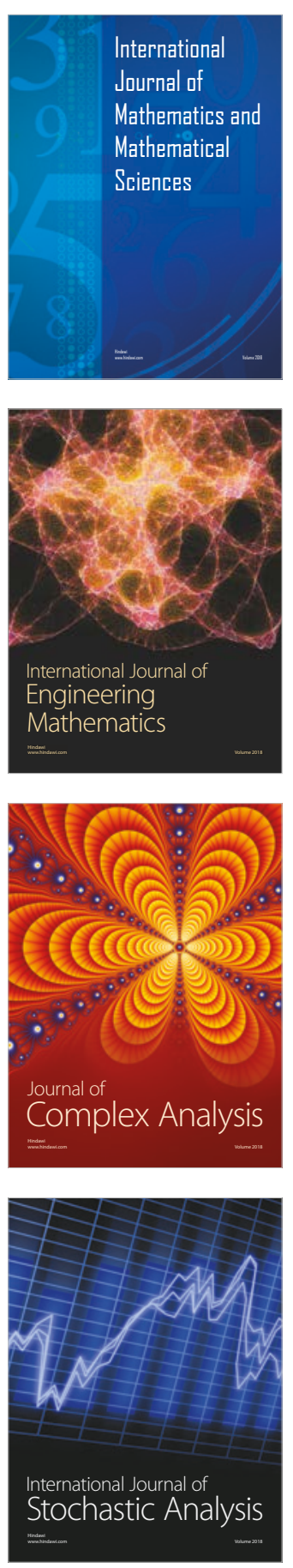
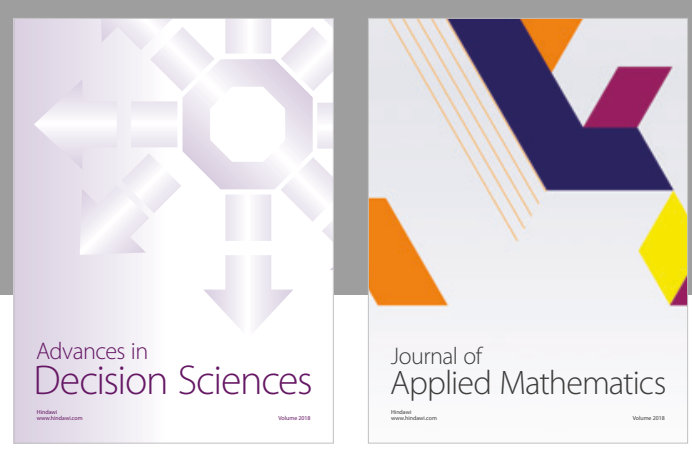

Journal of

Applied Mathematics
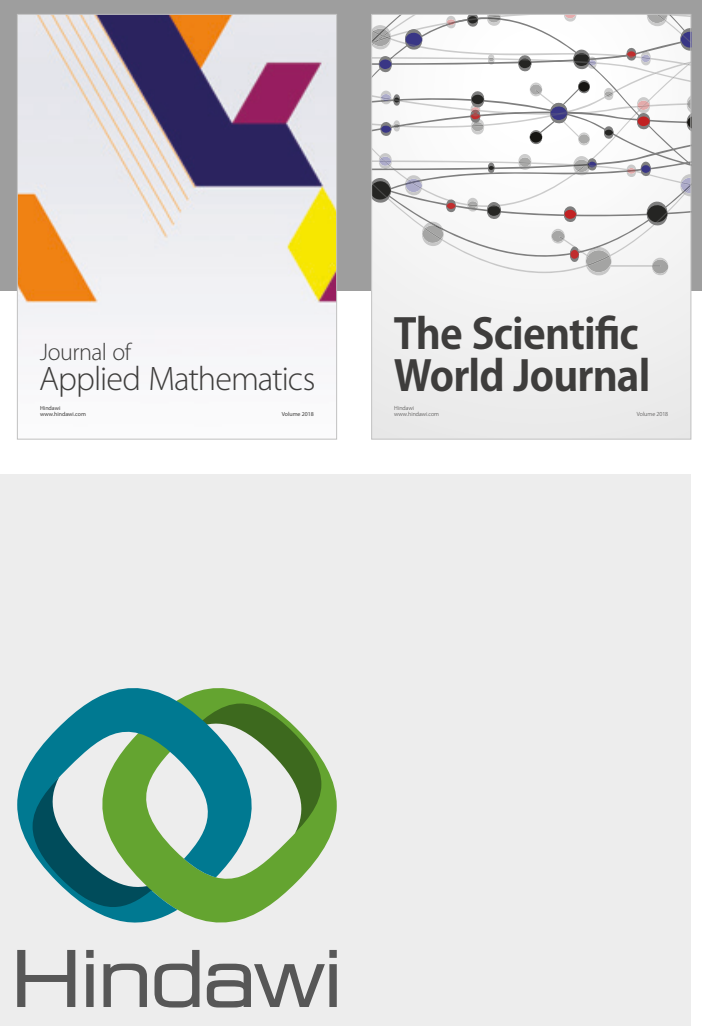

Submit your manuscripts at

www.hindawi.com

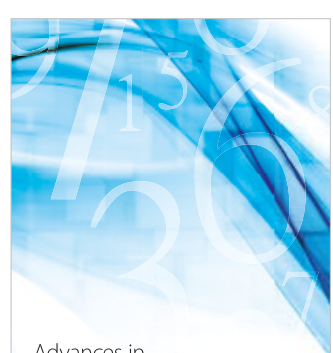

Advances in
Numerical Analysis
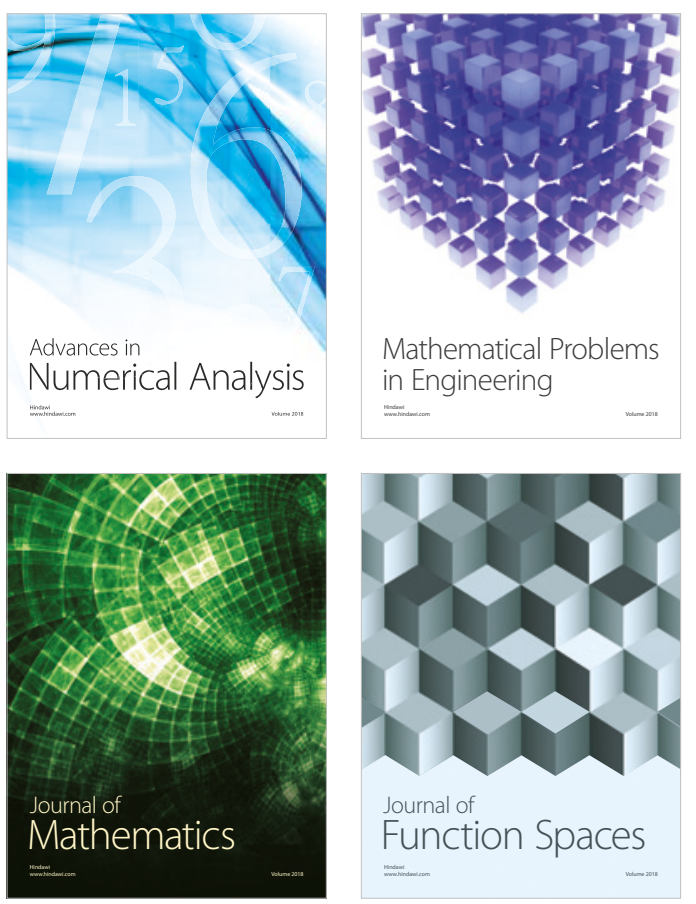

Mathematical Problems in Engineering

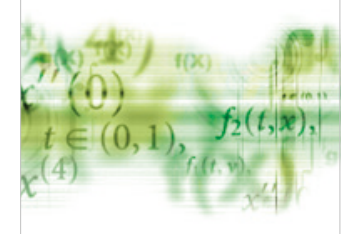

International Journal of

Differential Equations

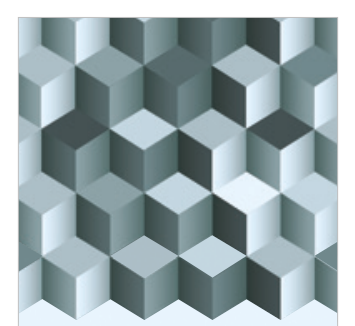

Journal of

Function Spaces
The Scientific

World Journal

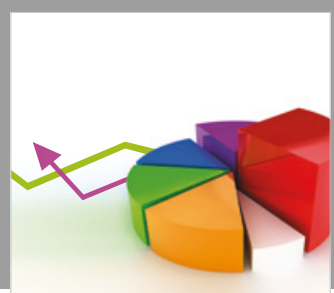

Journal of

Probability and Statistics
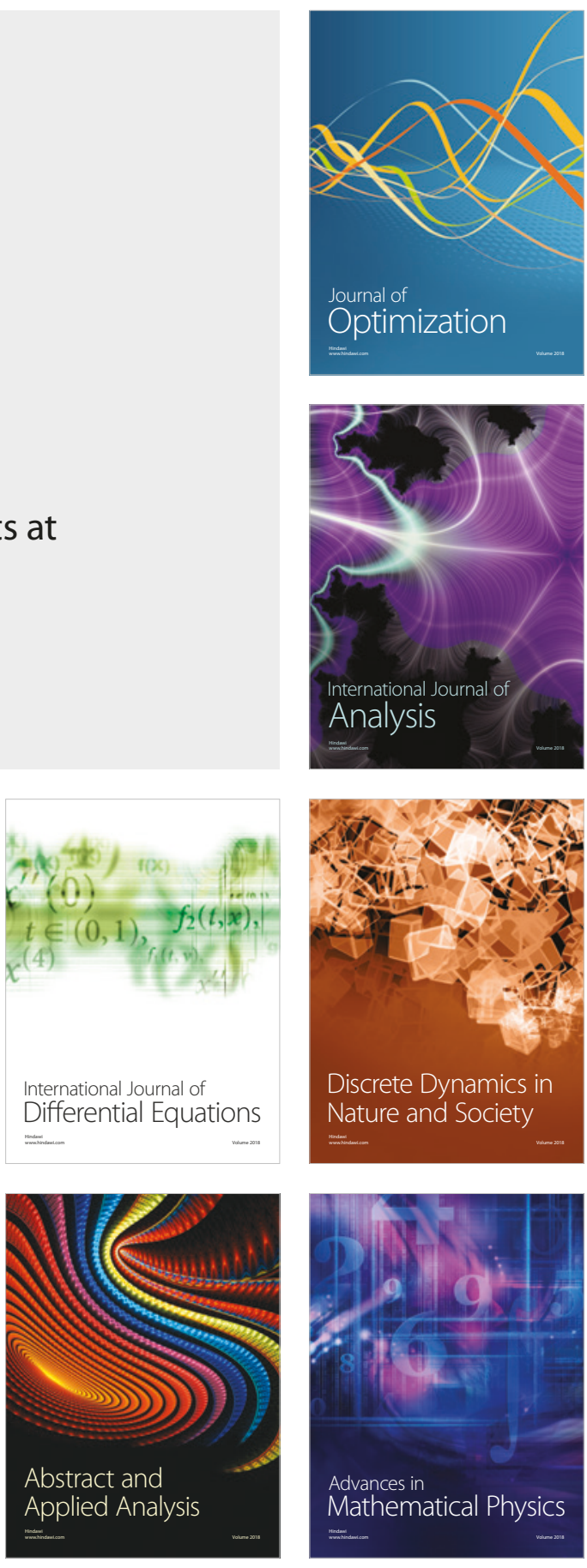